\title{
CMEARTICLE
}

\section{Clinics in diagnostic imaging (207)}

Jia Ren Perry Liew ${ }^{1}$, MBBS, FRCR, Yurui David Lim ${ }^{1}$, MBBS, FRCR, Jin Yee Charlene Liew ${ }^{1}$, MBBS, FRCR, Choo Choo Angeline $\underline{\mathrm{Poh}}^{1}$, MBBS, FRCR

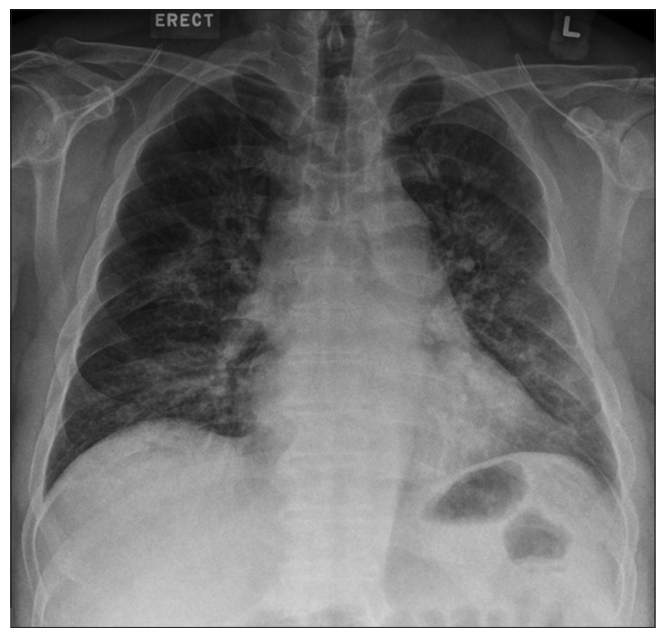

Fig. 1 Chest radiograph taken on Day 1 of admission.

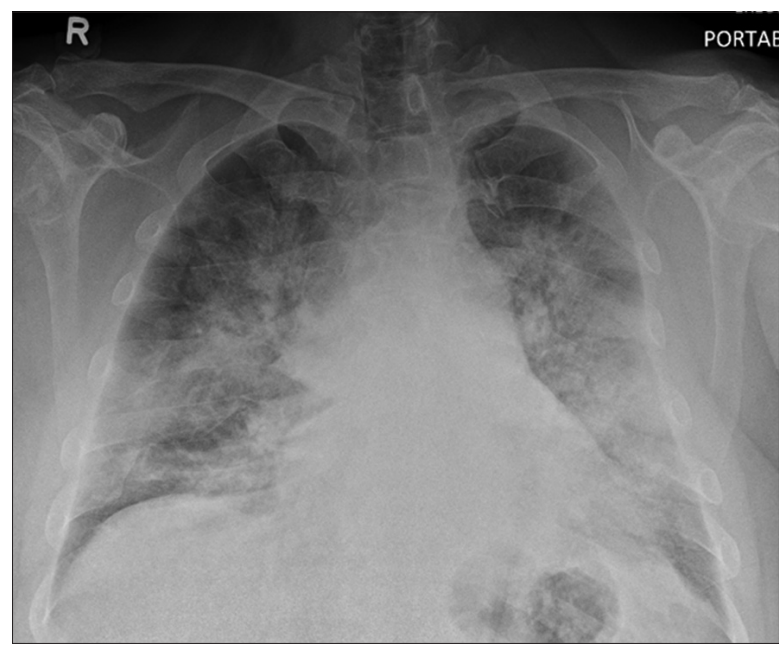

Fig. 2 Chest radiograph taken on Day 2 of admission.

\section{CASE PRESENTATION}

A 53-year-old Chinese man with a past medical history of metabolic syndrome and spinal spondylosis presented with a one-week history of high fever and non-productive cough. The symptoms did not resolve despite the patient having completed a course of antibiotics given by his general practitioner. He did not have recent travel or contact history with anyone with chest infection. He was febrile with a temperature of $39.1^{\circ} \mathrm{C}$ and had crepitations of both the middle and lower zones on lung auscultation. He was saturating well on room air and his other vital signs were normal. Initial blood tests showed a total white blood cell count of $10.4 \times 10^{3} / \mathrm{dL}$. C-reactive protein was raised at $199 \mathrm{mg} / \mathrm{L}$. He was initially treated for community-acquired pneumonia, pending microbiological tests. What do the chest radiographs at admission (Fig. 1) and on the following day (Fig. 2) show? 

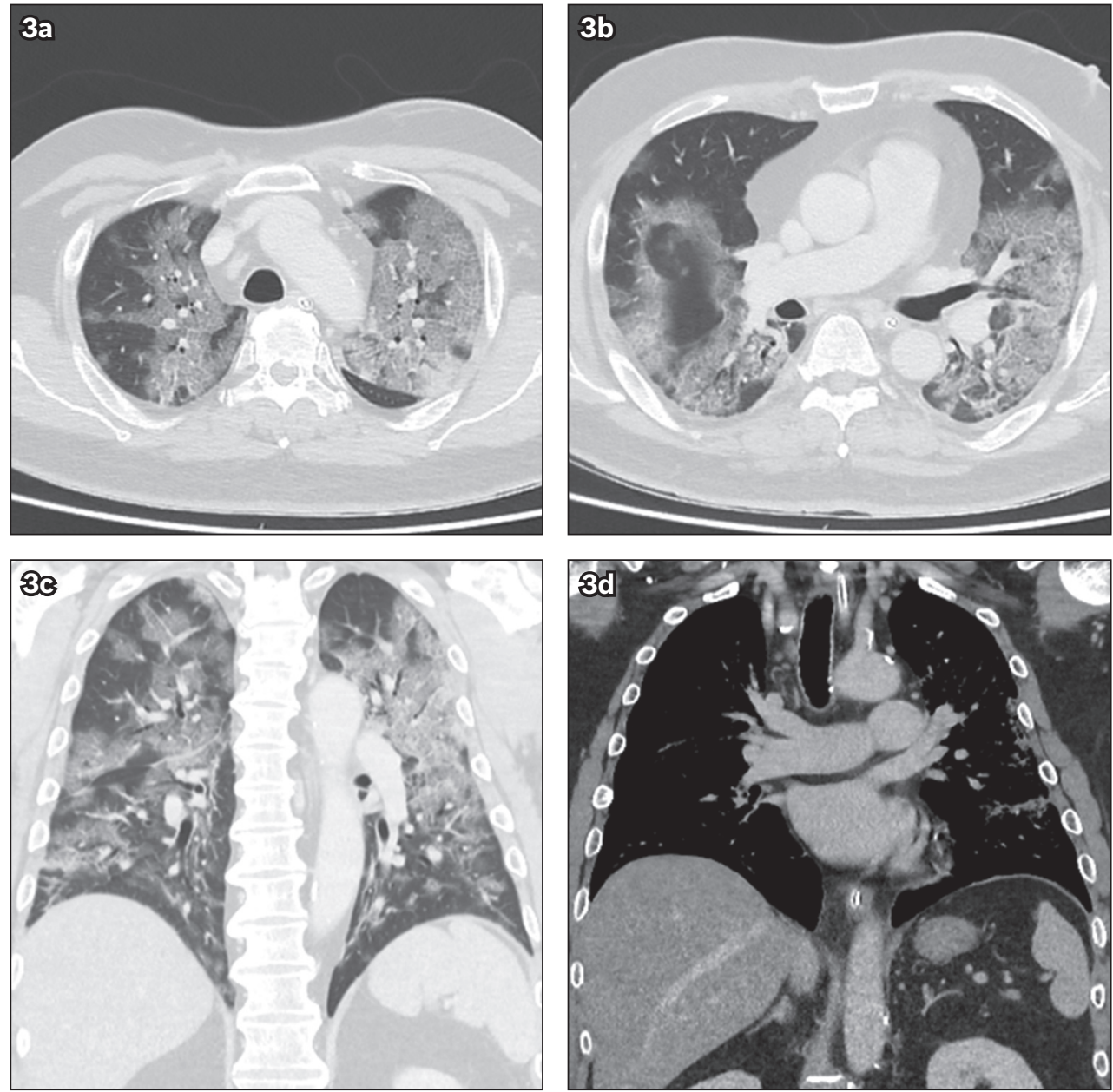

Fig. 3 (a \& b) Axial and (c) coronal chest CT images obtained using lung windows, and (d) coronal images obtained using soft tissue windows.

Shortly after admission, the patient deteriorated and was intubated. The total white blood cell count increased to $17.0 \times 10^{3} / \mathrm{dL}$. Initial Gram stain and culture from the endotracheal tube showed Klebsiella pneumoniae. The patient was started on antibiotic therapy comprising intravenous (IV) co-amoxiclav $1.2 \mathrm{~g}$ eight-hourly, which was subsequently escalated to IV Tazocin 4.5 g eight-hourly. Subsequently, his oxygen requirements decreased and he was extubated. However, he continued to have high temperature spikes. To investigate for other possible sources of sepsis, he underwent computed tomography (CT) on Day 5 of admission. What does the CT of the chest (Fig. 3) show?

\section{IMAGE INTERPRETATION}

The initial chest radiograph on presentation (Fig. 1) shows patchy air space opacities in both the middle and lower zones. Together with the clinical history of infective symptoms, the radiographic diagnosis was pulmonary infection. The lung changes worsened on the chest radiograph taken one day later (Fig. 2), increasing in density and extent of involvement to include both the upper and lower zones. Chest CT showed multifocal confluent ground-glass opacities and mixed ground-glass with patchy consolidation in both lungs, optimally seen on lung window settings (Figs. 3a-c). There was thickening of the interlobular septa on a background of ground-glass opacities, reminiscent of a 'crazy paving' appearance. No cavitating lesion or pleural effusion was seen.
No enlarged mediastinal node was detected on CT soft tissue window settings (Fig. 3d).

\section{DIAGNOSIS}

Coronavirus disease 2019 (COVID-19) atypical pneumonia.

\section{CLINICAL COURSE}

The patient tested positive for COVID-19 and was subsequently isolated. He completed a regime of Tazocin 4.5 g eight-hourly for a week to treat the Klebsiella infection. He was also started on antiviral therapy of two Kaletra (lopinavir 200 mg, ritonavir $50 \mathrm{mg}$ ) tablets twice daily. He improved clinically and tested swab negative on Days 9 and 10 of admission. Further radiography on Day 13 (Fig. 4a) and Day 18 (Fig. 4b) showed interval improvement and resolution of the bilateral lung opacities, respectively. The patient made a full recovery and was discharged on Day 16.

\section{DISCUSSION}

The radiological finding of 'crazy paving' on chest CT itself is non-specific and can be seen in several conditions. Differential diagnosis includes atypical pneumonia, acute respiratory distress syndrome, pulmonary alveolar proteinosis and non-specific interstitial pneumonia. ${ }^{(1)}$ Atypical pneumonia may be caused by a wide range of infections, including viral pneumonia. 

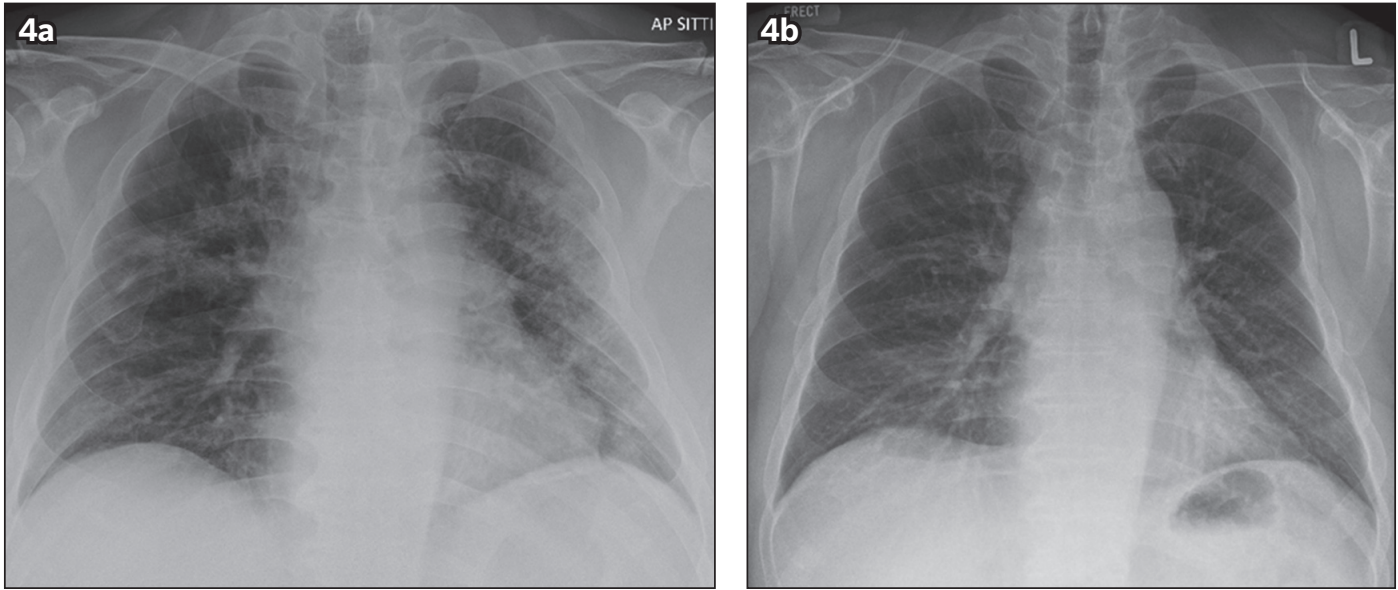

Fig. 4 Chest radiograph taken on (a) Day 13 of admission shows interval improvement of bilateral lung opacities; and (b) Day 18 of admission shows resolution of bilateral lung opacities.

In the current pandemic, COVID-19 is an important differential diagnosis that needs to be considered in all patients presenting with chest infection. The typical CT findings of COVID-19 patients in China have been described during the initial period of the pandemic, consisting of predominant groundglass opacities in the peripheries and usually the posterior aspects of the lungs. Approximately one-third of the patients also had pulmonary consolidation. ${ }^{(2-4)}$

Ground-glass opacities refer to the presence of opacities on $\mathrm{CT}$ in which the outlines of the blood vessels can still be seen. Consolidations are further increased in the density of such opacities, such that the blood vessels are no longer visible. There is also a lack of cavitating nodules, pleural effusion or enlarged mediastinal lymph node. ${ }^{(2-4)}$ In another patient with COVID-19 pneumonia of lesser severity (Fig. 5), CT showed predominantly peripheral groundglass changes with consolidation, more in the posterior aspects of the lungs. No 'crazy paving' appearance was seen for this patient. Similarly, no cavitating lesion or pleural effusion was detected.

In a series of 81 COVID-19 patients, Shi et al reported a slight predominance of the right lower lobe but indicated that all lobes could be involved. There was progressive increase of consolidation with increased duration of the infection. A small proportion of patients showed pleural effusion, cystic change and lymphadenopathy in the third week from symptom onset. Although the presence of pleural effusion may be a bad prognostic factor, it is also seen in other viral pneumonias. ${ }^{(5)}$

In comparison with the chest CT of patients with severe acute respiratory syndrome (SARS), another coronavirus, it is not surprising that the features are largely similar. CT thorax findings of SARS are also classically described as peripheral, ground-glass opacities, sometimes with consolidation. ${ }^{(6)}$ Another atypical infection that needs to be considered, especially if the patient is immunocompromised, is Pneumocystis pneumonia. It is an opportunistic yeast infection that occurs when CD4 T lymphocyte levels fall below 200 cell/ $\mu \mathrm{L}$. Typical radiographic findings include bilateral perihilar opacities. CT can show ground-glass opacities in the perihilar central regions, with some demonstrating reticular changes or a 'crazy paving' appearance, and subpleural sparing.(7) A small proportion of patients will have granulomatous changes, including enlarged mediastinal lymph nodes, cavitating nodules or pleural effusion. (7) In our patient, the distribution of the ground-glass opacities was not distinctively perihilar.

Acute respiratory distress syndrome is an acute lung injury characterised by non-cardiogenic pulmonary oedema and lung inflammation. Patients have impaired lung diffusion capacity, respiratory distress and hypoxaemia. Due to its non-cardiogenic nature, radiographs show bilateral, largely symmetrical opacities with the lack of upper lobe diversion and Kerley B lines (thin septal lines seen at the periphery of the lungs on chest radiographs) ${ }^{(8)}$ (Fig. 6a). CT findings include bilateral, basal predominant ground-glass opacities and consolidation with increased reticular markings ${ }^{(8)}$ (Figs. 6b-c). Worsening changes may include bronchiectasis and honeycombing. Cysts may also develop during the course of the disease. ${ }^{(8)}$ As the ground-glass changes in our patient involve the upper lobes as well, this differential is less likely.

Pulmonary alveolar proteinosis is a process of intra-alveolar accumulation of lipoproteinaceous material. It is predominantly autoimmune in aetiology, with other lesser aetiologies, including being secondary to haematological malignancy or inhalational lung disorders, or congenital in nature. ${ }^{(9)}$ Chest CT shows the classical description of a 'crazy paving' appearance with diffuse ground-glass opacities and interlobular septal thickening. These changes have a lower lobe predisposition. ${ }^{(9)}$

Non-specific interstitial pneumonia is a type of interstitial lung disease with interstitial fibrosis and inflammation. Radiological features include the predominance of basal ground-glass opacities with interstitial thickening, traction bronchiectasis, fibrosis and lower lobe volume loss ${ }^{(10)}$ (Figs. 7a-c). Traction bronchiectasis is seen when there is an enlargement of the airway at the periphery of the lung, associated with adjacent scarring. However, the temporal evolution of $\mathrm{CT}$ changes is rather chronic in nature, and its time course does not fit our patient's presentation.

Klebsiella pneumonia has different features on $\mathrm{CT}$ imaging. In Okada et al's series, all the cases of Klebsiella pneumonia had ground-glass opacities and intralobular septal thickening, with a much higher proportion of consolidation. The disease process occurred predominantly in the lower zones, although 

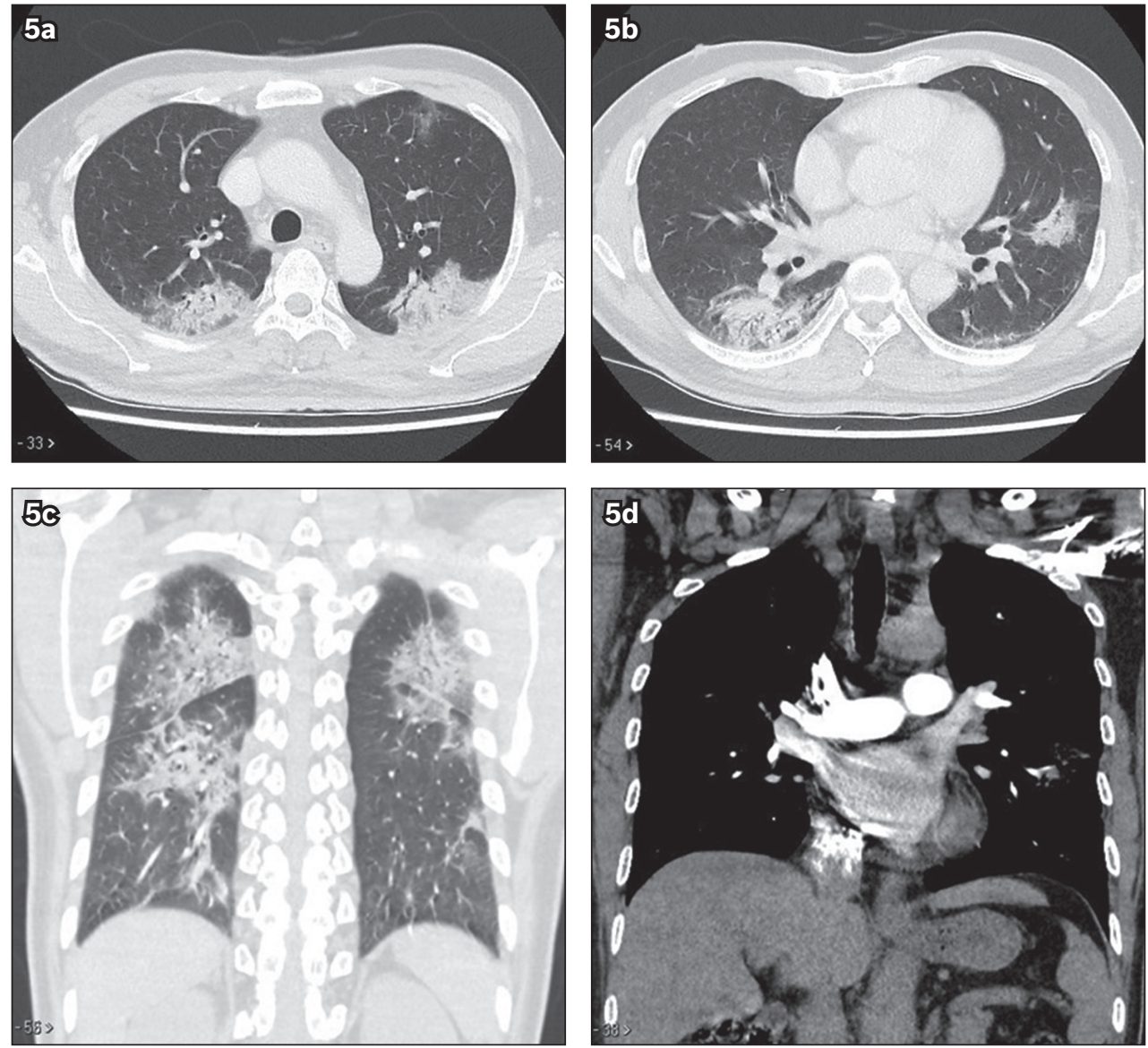

Fig. 5 CT thorax of another patient with coronavirus disease 2019. (a \& b) Axial, (c) coronal lung window and (d) coronal soft tissue window images show multifocal confluent ground-glass changes and mixed ground-glass opacities with patchy consolidation in both lungs. Note the lack of cavitating nodules, enlarged mediastinal nodes and pleural effusion.
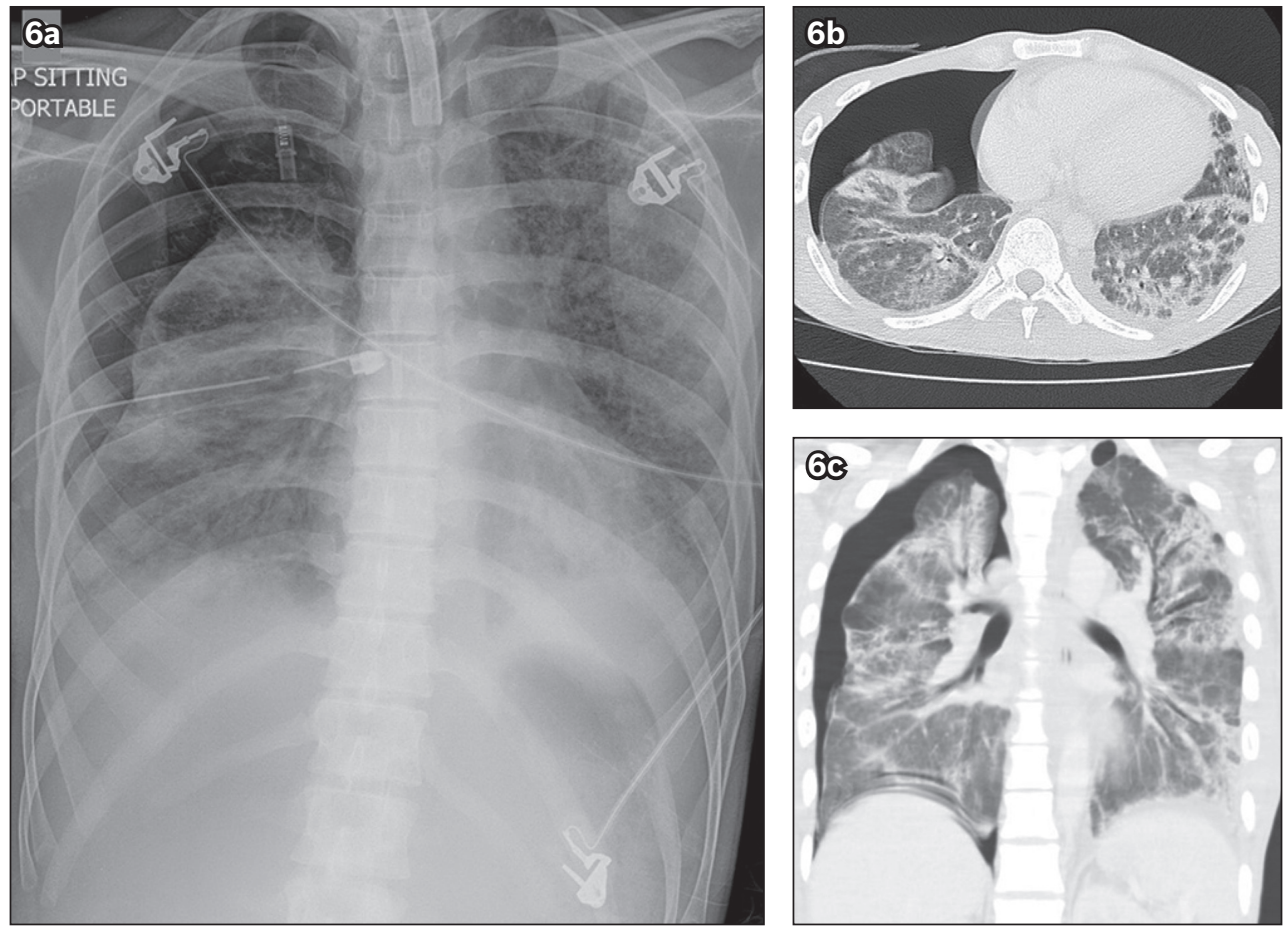

Fig. 6 A patient with acute respiratory distress syndrome. (a) Chest radiograph shows bilateral symmetric pulmonary opacities with a lack of upper lobe diversion. Tracheostomy, right-sided chest tube and pneumothorax are present. (b) Axial and (c) coronal chest CT thorax images show bilateral basal predominant ground-glass opacities and consolidation with increased reticular markings. These features are typical of acute respiratory distress syndrome. 

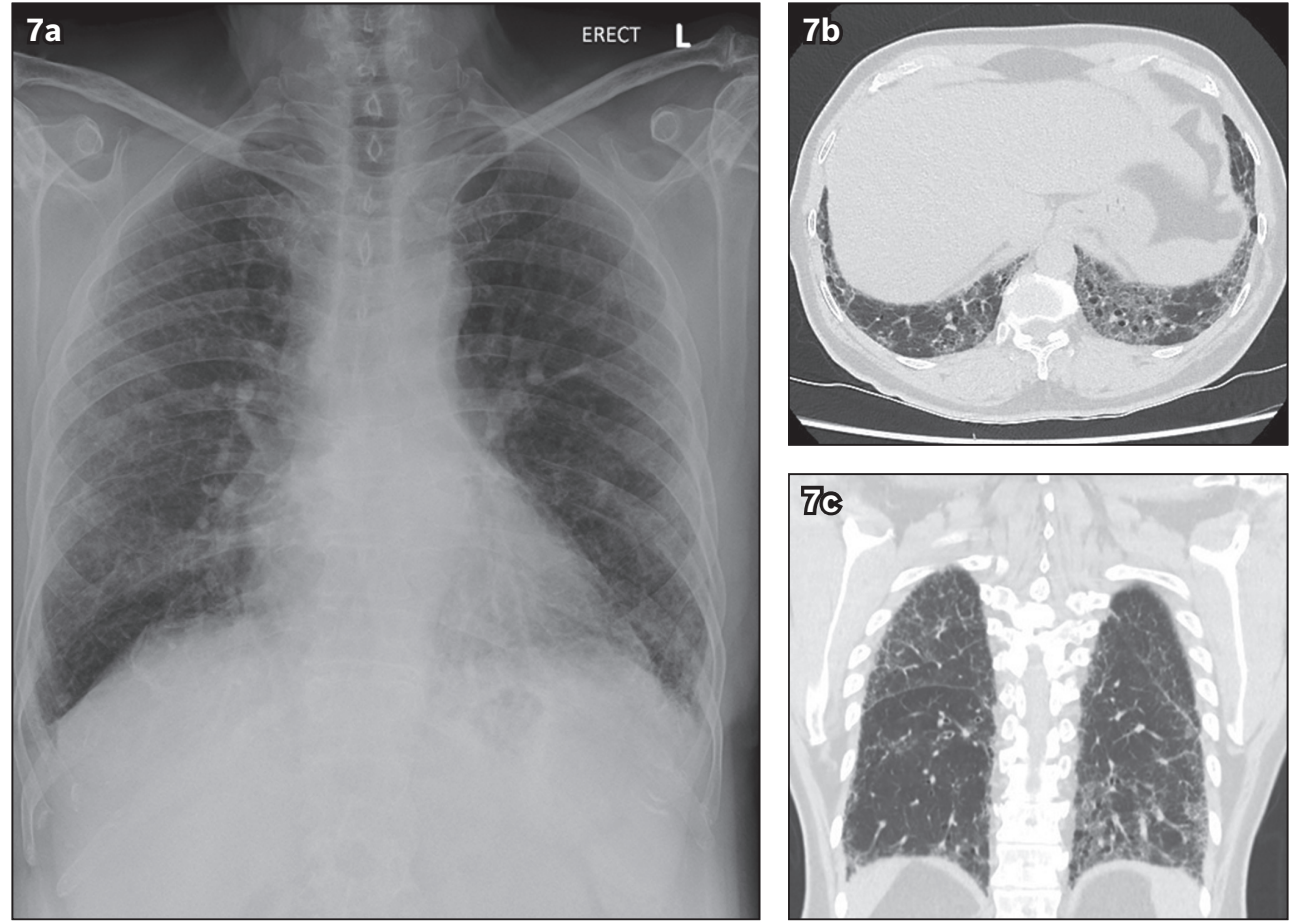

Fig. 7 A patient with non-specific interstitial pneumonia. (a) Chest radiograph shows peripheral and basal predominant reticular markings. (b) Axial and (c) coronal chest CT thorax images show basal predominant interstitial thickening, traction bronchiectasis, ground-glass opacities and lower lobe volume loss.

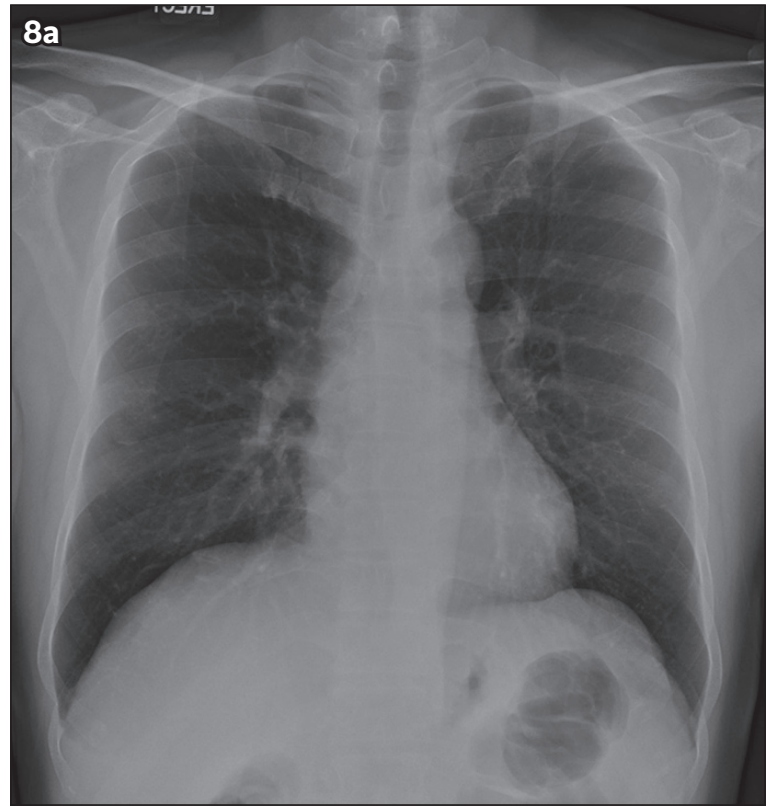

Fig. 8 Serial chest radiographs of another patient with COVID-19 infection of lesser severity. (a) Day 1 chest radiograph is normal. (b) Day 11 chest radiograph shows bilateral mid-zone opacities, in keeping with infective changes. (c) Day 17 chest radiograph shows interval improvement of the bilateral mid-zone opacities.
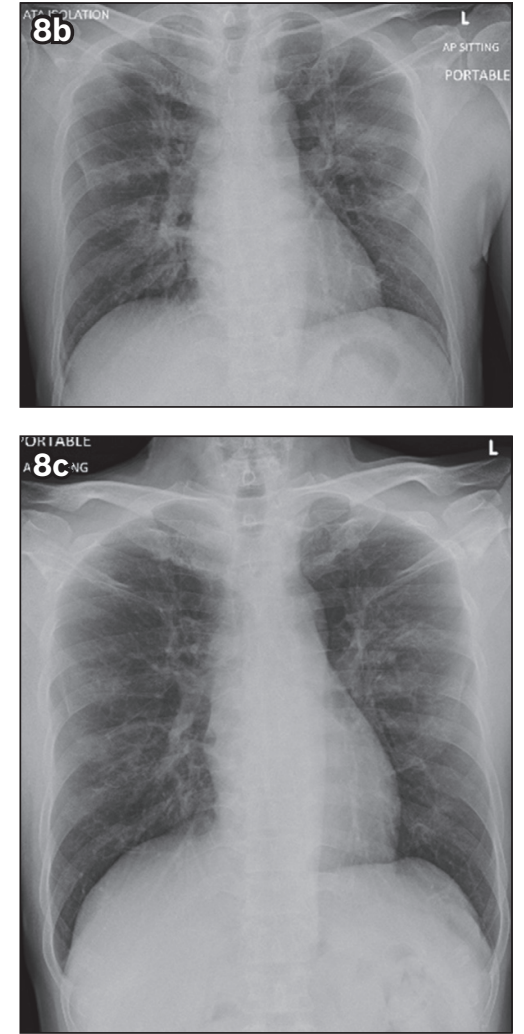

it was also peripheral in nature. Effusions were seen in half the patients and enlarged nodes were seen in a small proportion of patients. ${ }^{(11)}$ The bulging fissure sign is classically used to describe hospital-acquired Klebsiella pneumonia infection with large amounts of infective fluid within the affected right upper lobe. ${ }^{(12)}$ Air-fluid level sign is also a radiographic description of air within an abscess that can occur in Klebsiella pneumonia infection. ${ }^{(12)}$
In our experience with COVID-19 patients, the progression of the patients' radiographs (Figs. 1, $2 \& 4$ for the first patient and Fig. 8 for the second patient) corresponds to a progression pattern that is described in a prior paper on SARS. Wong et al described a predominant Type 1 pattern in which patients deteriorated until a peak level before having radiographic improvement of opacities. ${ }^{(6)}$ This was mirrored in our two COVID-19 patients. 
In SARS, the duration from diagnosis to deterioration of imaging findings ranged from two to eight days, ${ }^{(6)}$ while in our two patients, deterioration of imaging findings was seen from one to 11 days. By comparison, the published literature shows a wider range of progression, from no progression for up to eight days to worsening in 3-4 days. ${ }^{(3)}$ The differences could be due to a lack of daily radiographs, the presence of co-infection in the first patient, and the small sample size that our paper describes.

From the current published medical knowledge, it seems that COVID-19 infection can occur in asymptomatic individuals with a long incubation period. ${ }^{(13)}$ The gold standard for diagnosing infection by reverse transcription polymerase chain reaction is currently limited, due to a set turnaround time and availability of test kits. Radiographic imaging is useful for detection of pneumonia in unsuspected cases, such as in the first patient. Kim suggested that imaging could play other roles in patient management, including disease severity triaging, deterioration prediction and detection of superimposed infection. ${ }^{(14)}$

Currently, the American College of Radiology cautions against the role of CT in diagnosing COVID-19 but notes that radiologists need to be familiar with the features of COVID-19 infection in patients who are imaged for other reasons. ${ }^{(15)} \mathrm{A}$ consensus statement by the Radiology Society of North America recommends a standardised format of reporting chest CT findings with regard to COVID-19, classifying findings into typical appearances, intermediate appearances, atypical appearances and negative for pneumonia. This will benefit downstream management and avoid unnecessary panic or confusion due to non-standardised terminology. ${ }^{(16)}$

With the use of deep learning techniques, Lin et al was able to demonstrate high accuracy and specificity in the diagnosis of COVID-19 from community-acquired pneumonia, although the authors recognise the inherent lack of transparency in current deep learning techniques and the lack of specificity of certain lung imaging findings without a multidisciplinary approach. ${ }^{(17)}$ Lum et al mentioned the need for a united worldwide endeavour in improving diagnostics, research toward an effective cure, population surveillance and social measures to counter the disease. ${ }^{(18)}$ We have great hopes that through cohesive efforts and increased vigilance, we will be able to overcome this epidemic.

In summary, COVID-19 is an infection that may be difficult to detect due to its long incubation time and reports of its presence in asymptomatic individuals. Imaging can aid in the diagnosis of COVID-19 with the hallmark features of bilateral peripheral ground-glass opacities, with a proportion of patients having consolidative changes and 'crazy paving' appearance. In the published literature to date, findings of enlarged nodes, cavitating nodules and pleural effusions are not the typical findings for COVID-19. It is important for radiologists to raise the suspicion of atypical pneumonia in such circumstances to enable early patient isolation and limit the spread of infection.

\section{ACKNOWLEDGEMENT}

We wish to acknowledge Dr Philip Leong for reporting the chest CT of one of the cases.

\section{REFERENCES}

1. Rossi SE, Erasmus JJ, Volpacchio M, et al. "Crazy-paving" pattern at thin-section CT of the lungs: radiologic-pathologic overview. Radiographics 2003; 23:1509-19.

2. Kanne JP. Chest CT findings in 2019 novel coronavirus (2019-nCoV) infections from Wuhan, China: key points for the radiologist. Radiology 2020; 295:16-7.

3. $\mathrm{Ng}$ MY, Lee EY, Yang J, et al. Imaging profile of the COVID-19 infection: radiologic findings and literature review. Radiol Cardiothorac Imaging [online]. Available at: http://pubs.rsna.org/doi/10.1148/ryct.2020200034. Accessed February 26, 2020.

4. Chung M, Bernheim A, Mei X, et al. CT imaging features of 2019 novel coronavirus (2019-nCoV). Radiology 2020; 295:202-7.

5. Shi H, Han X, Jiang N, et al. Radiological findings from 81 patients with COVID-19 pneumonia in Wuhan, China: a descriptive study. Lancet Infect Dis 2020; 20:425-34

6. Wong KT, Antonio GE, Hui DS, et al. Severe acute respiratory syndrome: radiographic appearances and pattern of progression in 138 patients. Radiology 2003; 228:401-6.

7. Afione C, Della Sala A, Frank L. Pulmonary manifestations in patients with AIDS. RAR 2011; 75:1-14. Available at: https://www.webcir.org/revistavirtual/ articulos/marzo12/argentina/arg_ingles.pdf. Accessed March 31, 2020.

8. Sheard S, Rao P, Devaraj A. Imaging of acute respiratory distress syndrome. Respir Care 2012; 57:607-12.

9. Frazier AA, Franks TJ, Cooke EO, et al. From the archives of the AFIP: pulmonary alveolar proteinosis. Radiographics 2008; 28:883-99.

10. Kligerman SJ, Groshong S, Brown KK, Lynch DA. Nonspecific interstitial pneumonia: radiologic, clinical, and pathologic considerations. Radiographics 2009; 29:73-87.

11. Okada F, Ando Y, Honda K, et al. Clinical and pulmonary thin-section CT findings in acute Klebsiella pneumoniae pneumonia. Eur Radiol 2008; 19:809-15.

12. Walker CM, Abbott GF, Greene RE, et al. Imaging pulmonary infection: classic signs and patterns. AJR Am J Roentgenol 2014; 202:479-92.

13. Bai $Y$, Yao $L$, Wei $T$, et al. Presumed asymptomatic carrier transmission of COVID-19. JAMA 2020; 323:1406-7.

14. Kim H. Outbreak of novel coronavirus (COVID-19): what is the role of radiologists? Eur Radiol 2020; 30:3266-7.

15. American College of Radiology. ACR recommendations for the use of chest radiography and computed tomography $(C T)$ for suspected COVID-19 infection. Available at: https://www.acr.org/Advocacy-and-Economics/ACR-PositionStatements/Recommendations-for-Chest-Radiography-and-CT-for-SuspectedCOVID19-Infection. Accessed March 26, 2020.

16. Simpson S, Kay FU, Abbara S, et al. Radiological Society of North America Expert Consensus Statement on reporting chest CT findings related to COVID-19. Endorsed by the Society of Thoracic Radiology, the American College of Radiology, and RSNA. Radiology Cardiothorac Imag 2020 Mar 25. https://doi. org/10.1148/ryct.2020200152.

17. Li L, Qin L, Xu Z, et al. Artificial intelligence distinguishes COVID-19 from community acquired pneumonia on chest CT. Radiology 2020 Mar 19. https:// doi.org/10.1148/radiol.2020200905. [Epub ahead of print]

18. Lum LHW, Tambyah PA. Outbreak of COVID-19 - an urgent need for good science to silence our fears? Singapore Med J 2020; 61:55-7. 


\section{SINGAPORE MEDICAL COUNCIL CATEGORY 3B CME PROGRAMME} (Code SMJ 202007B)

Question 1. Based on the latest published literature regarding the coronavirus disease 2019 (COVID-19) pandemic, what are the typical features of COVID-19 infection on computed tomography (CT) of the chest during the early stages of infection?
(a) Ground glass opacities in a peripheral distribution.
(b) Pulmonary consolidation with tree-in-bud changes.
(c) Ground glass opacities in a perihilar distribution with cystic changes.
(d) Cavitating nodules with pleural effusion.

Question 2. What are other possible differential diagnoses for a 'crazy paving' appearance?
(a) Pneumocystis pneumonia.
(b) Non-specific interstitial pneumonia.
(c) Klebsiella pneumonia.
(d) Acute respiratory distress syndrome.

Question 3. How are the imaging findings of COVID-19 pneumonia different from those of Klebsiella pneumonia? Chest CT findings of patients with COVID-19 pneumonia tend to have a:
(a) Higher proportion of pulmonary lobar consolidation.
(b) Higher proportion of mediastinal lymphadenopathy.
(c) Higher proportion of pleural effusion.
(d) Higher proportion of cavitary nodules.

Question 4. Which of the following imaging features can help to differentiate the diagnosis of cardiogenic pulmonary oedema from COVID-19 pneumonia?

(a) Bilateral pleural effusions.

(b) Presence of pulmonary upper lobe venous diversion.

(c) Presence of 'crazy paving' opacities in the case of COVID-19 pneumonia.

(d) Kerley B lines.

Question 5. Regarding the role of radiology in the diagnosis of COVID-19 infection:

(a) A normal chest radiograph excludes COVID-19 infection.

(b) Radiographic imaging, such as chest radiography and CT, can aid in the detection of COVID-19 pneumonia in unsuspected cases.

(c) $\mathrm{CT}$ of the chest is now considered the gold standard for diagnosis.

(d) Other roles of imaging in patient management during the COVID-19 pandemic include triaging of disease severity and survivorship, as well as the management of complications.

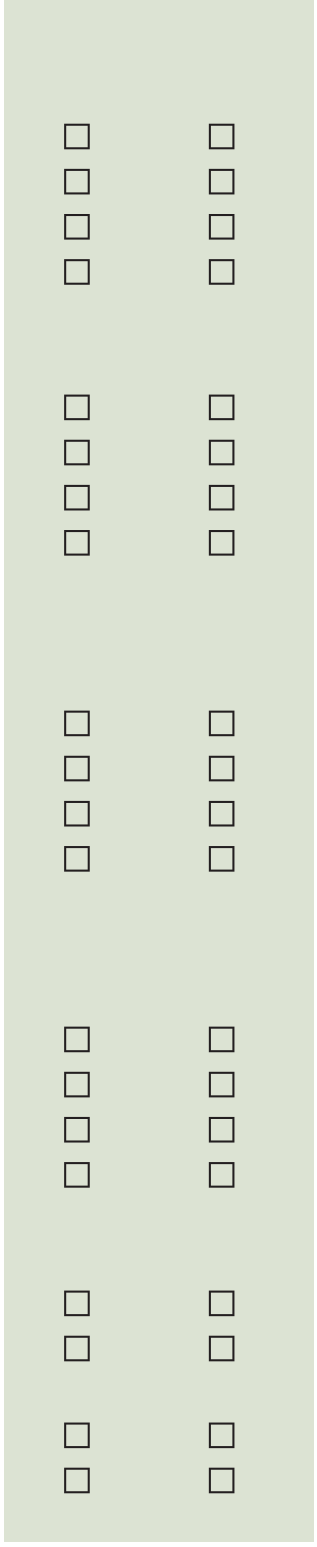

\footnotetext{
Doctor's particulars:

Name in full:

MCR no.:

Specialty:

Email:

SUBMISSION INSTRUCTIONS:

Visit the SMJ website: http://www.smj.org.sg/current-issue and select the appropriate quiz. You will be redirected to the SMA login page.

For SMA member: (1) Log in with your username and password (if you do not know your password, please click on 'Forgot your password?'). (2) Select your answers for each quiz and click 'Submit'.

For non-SMA member: (1) Create an SMJ CME account, or log in with your SMJ CME username and password (for returning users). (2) Make payment of SGD 21.40 (inclusive of $7 \%$ GST) via PayPal to access this month's quizzes. (3) Select your answers for each quiz and click 'Submit'.

RESULTS:

(1) Answers will be published online in the SMI September 2020 issue. (2) The MCR numbers of successful candidates will be posted online at the SMI website by 10 September 2020. (3) Passing mark is $60 \%$. No mark will be deducted for incorrect answers. (4) The SMJ editorial office will submit the list of successful candidates to the Singapore Medical Council. (5) One CME point is awarded for successful candidates. (6) SMC credits CME points according to the month of publication of the CME article (i.e. points awarded for a quiz published in the June 2020 issue will be credited for the month of June 2020, even if the deadline is in August 2020).

Deadline for submission (July 2020 SMJ 3B CME programme): 12 noon, 3 September 2020.
} 